\title{
Revealing the true face of the behemoths
}

\author{
Keiichi Ohnaka \\ Universidad Católica del Norte, Instituto de Astronomía, \\ Avenida Angamos 0610, Antofagasta, Chile \\ email: k1.ohnaka@gmail.com
}

\begin{abstract}
To improve our understanding of the mass loss in red supergiants (RSGs), it is of paramount importance to observe the wind acceleration region close to the star with high spatial resolution. I review the recent progress in milliarcsecond-resolution imaging of the surface and atmosphere of RSGs with optical and infrared long-baseline interferometers. The high resolution images of RSGs have revealed the true face of these behemoths, which have turned out to be very complex.
\end{abstract}

Keywords. stars: atmospheres, stars: imaging, stars: late-type, stars: mass loss, (stars:) supergiants, infrared: stars, techniques: high angular resolution, techniques: interferometric

\section{Introduction}

Massive stars experience intensive mass loss in the red supergiant (RSG) phase, which significantly affects the evolution of the stars themselves. For example, the RSG mass loss is considered to be a key to constraining the mass of the progenitors of supernovae (SNe) type IIP, which are the most common core-collapse SNe (e.g., Maund 2015 in this volume). Despite such importance, the mass loss from RSGs is one of the long-standing problems in stellar astrophysics.

To address this problem, it is crucial to spatially resolve the region between the photosphere and the innermost circumstellar envelope, where the mass outflows are considered to be accelerated. However, the angular scale of this region (a few stellar radii from the star) is very small. For example, the closest RSG Betelgeuse has an angular diameter of 42.5 mas (Ohnaka et al. 2011). This angular diameter is much smaller than the angular resolution of conventional ground-based imaging of $\sim 1^{\prime \prime}$, which is limited by seeing. With adaptive optics, it is possible to achieve angular resolution much closer to the diffraction limit of the telescope, which is 30 mas at $1 \mu \mathrm{m}$ with an $8 \mathrm{~m}$ telescope. While the innermost circumstellar envelope (within $\sim 10$ stellar radii) of Betelgeuse can be well resolved with this resolution (Kervella et al. 2009), the surface of the star is only partially resolved. We need milliarcsecond resolution to spatially resolve the detailed structures of the wind acceleration region and the stellar surface of RSGs. Optical and infrared long-baseline interferometry provides the unique opportunity to achieve this scientific goal.

\section{Aperture-synthesis imaging of RSGs}

Haubois et al. (2009) obtained an aperture-synthesis image of Betelgeuse at $1.6 \mu \mathrm{m}$ with the Infrared Optical Telescope Array (IOTA). They detected two spots, which have a size of $1 / 4-1 / 2$ of the radius of the star with an intensity contrast of $5-10 \%$. Baron et al. (2014) presented the $1.65 \mu \mathrm{m}$ aperture-synthesis images of two RSGs, T Per and RS Per, in the Double Cluster obtained with the Center for High-Angular Resolution Astronomy (CHARA) array. While T Per shows a bright spot with a size comparable to the stellar radius, RS Per shows a large dark region near the limb. The contrast of 
the surface structures is approximately $15 \%$ in both cases. These spots are considered to represent large convective cells, which were predicted by Schwarzschild (1975) and modeled by 3D convection simulations (e.g., Chiavassa et al. 2010).

These RSGs are optically bright, which means that they do not have optically thick dust envelopes. Some RSGs, however, are enshrouded in a thick dust envelope, and one of such objects is VY CMa. The diffraction-limited aperture-masking observations with the Keck telescope show elongated, one-sided emission in the South of the star (Monnier et al. 1999). More recently, Monnier et al. (2014) have presented images of VY CMa reconstructed at 1.61, 1.67, and $1.73 \mu \mathrm{m}$ from the data taken with the PIONIER instrument at ESO's Very Large Telescope Interferometer (VLTI). Using the same data, 10 different teams carried out the image reconstruction. The median images of the results of 10 teams reveal an extended atmosphere elongated in the E-W direction and two bright spots with a size of the stellar radius in the Eastern half of the stellar surface.

\section{Velocity-resolved aperture-synthesis imaging}

The aforementioned studies demonstrate the feasibility to obtain images of the inhomogeneous surface and atmosphere of RSGs. It is now possible to go one step farther: spatially resolving the dynamics (i.e., gas motions) of the atmosphere of stars, just as in Solar physics. Taking advantage of the high spectral resolution provided by the nearinfrared VLTI instrument AMBER, Ohnaka et al. (2011) presented 1D image reconstruction of Betelgeuse in the individual CO first overtone lines near $2.3 \mu \mathrm{m}$ with an angular resolution of 9.8 mas. The spectral resolution was high enough to have $\sim 10$ wavelength points within each $\mathrm{CO}$ line profile. The $1 \mathrm{D}$ images reconstructed in the blue wing and the line center of the CO line profiles show an atmosphere asymmetrically extending to $\sim 1.3$ stellar radii. However, the $1 \mathrm{D}$ images in the red wing of the $\mathrm{CO}$ lines do not show the extended component. The observed different appearance of the star across the CO line profiles can be explained by an inhomogeneous velocity field in the extended atmosphere. The modeling of Ohnaka et al. (2011) suggests vigorous upwelling and downdrafting motions of a gas clump as large as the radius of the star at velocities of up to $20-30 \mathrm{~km} \mathrm{~s}^{-1}$. Ohnaka et al. (2013) succeeded in spatially resolving a similar, inhomogeneous velocity field in another well-studied RSG, Antares, based on VLTI/AMBER data.

High spatial and high spectral resolution AMBER observations also allow us to extract the spatially resolved spectrum at each position over the surface of stars and in the atmosphere. The spatially resolved spectra from the extended atmosphere of Betelgeuse show prominent emission, while those inside the limb of the star show absorption, which is exactly what we expect (Ohnaka 2013). This means that we can map the 2D velocity field from the wavelength shifts of the spatially resolved spectra in a straightforward manner (Ohnaka et al. 2015, in prep).

The vigorous, inhomogeneous motions observed in Betelgeuse and Antares may be reminiscent of large convective cells. However, the density of the extended atmosphere at 1.3 stellar radii estimated from the observed data is $\sim 10^{-14} \mathrm{~g} \mathrm{~cm}^{-3}$, which is higher than that predicted by the current 3D convection simulations (Chiavassa et al. 2010) by 6 to 11 orders of magnitude. This suggests that convection alone cannot lift up the atmosphere to the observed 1.3 stellar radii. Arroyo-Torres et al. (2015) reached the same conclusion based on an "interferometric snapshot" survey for a small sample of RSGs. Furthermore, they found out that the atmospheric extension increases with the luminosity or decreasing surface gravity. On the other hand, the atmospheric extension shows no correlation with effective temperature or variability amplitude. Based on these 
results, they suggest that radiation pressure on molecular lines may drive the mass loss in RSGs.

\section{Prospects}

We can apply the velocity-resolved imaging to different atomic and molecular lines forming at different atmospheric heights, which allows us to obtain a $3 \mathrm{D}$ view of the atmospheric dynamics. This tomographic velocity-resolved imaging would be important for understanding how the energy and momentum propagate from the deep photosphere to the outer atmosphere, where they accelerate the stellar winds. High-resolution aperturesynthesis imaging is also important for directly probing the dust formation region. In particular, the second generation VLTI instrument MATISSE will start operations in near future (Lopez et al. 2014). This instrument will allow us to carry out aperturesynthesis imaging in the thermal infrared $(3-13 \mu \mathrm{m})$ for the first time, which is ideal for probing where and how dust forms. We can also study the chromosphere in great detail by high-spectral resolution interferometry. For example, Berio et al. (2011) spatially resolved the chromosphere of $\mathrm{K}$ giants in the $\mathrm{H} \alpha$ line and $\mathrm{Ca}$ II triplet lines. Similar studies for RSGs would be important for better understanding the structure and dynamics of the chromosphere. The combination of these high-angular resolution observations will provide us with a comprehensive picture of the complex atmosphere and circumstellar envelope of RSGs and help us solve the long-standing problem of the mass loss.

\section{References}

Arroyo-Torres, B., Wittkowski, M., Chiavassa, A., Scholz, M., Freytag, B., Marcaide, J. M., Hauschildt, P. H., Wood, P. R., \& Abellan, F. J. 2015, A\& $A$, 575, A50

Baron, F., Monnier, J. D., Kiss, L. L., Neilson, H. R., Zhao, M., Anderson, M., Aarnio, A., Pedretti, E., Thureau, N., ten Brummelaar, T. A., Ridgway, S. T., McAlister, H. A., Sturmann, J., Sturmann, L., \& Turner, N. 2014 ApJ, 785, 46

Berio, P., Merle, T., Thvenin, F., Bonneau, D., Mourard, D., Chesneau, O., Delaa, O., Ligi, R., Nardetto, N., Perraut, K., Pichon, B., Stee, P., Tallon-Bosc, I., Clausse, J. M., Spang, A., McAlister, H., ten Brummelaar, T., Sturmann, J., Sturmann, L., Turner, N., Farrington, C., \& Goldfinger, P. J. 2011, A\&SA, 535, A59

Chiavassa, A., Haubois, X., Young, J. S., Plez, B., Josselin, E., Perrin, G., \& Freytag, B. 2010, $A \mathscr{G} A, 515, \mathrm{~A} 12$

Chiavassa, A., Freytag, B., Masseron, T., \& Plez, B. 2011, A\& A, 535, A22

Haubois, X., Perrin, G., Lacour, S., Verhoelst, T., Meimon, S., Mugnier, L., Thiébaut, E., Berger, J. P., Ridgway, S. T., Monnier, J. D., Millan-Gabet, R., \& Traub, W. 2009, A\& A, 508, 923

Kervella, P., Verhoelst, T., Ridgway, S. T., Perrin, G., Lacour, S., Cami, J., \& Haubois, X. 2009, $A \mathscr{E} A, 504,115$

Lopez, B., Lagarde, S., \& Jaffe, W., et al. 2014, The Messenger, 157, 5

Monnier, J. D., Tuthill, P. G., Lopez, B., Cruzalebes, P., Danchi, W., \& Haniff, C. A. 1999, ApJ, 512,351

Monnier, J. D., Berger, J.-P., \& Le Bouquin, J.-B., et al. 2014, SPIE Proc, 9146, 91461Q

Ohnaka, K. 2013, EAS Publications Series, 60, 121

Ohnaka, K., Weigelt, G., Millour, F., Hofmann, K.-H., Driebe, T., Schertl, D., Chelli, A., Massi, F., Petrov, R., \& Stee, Ph. 2011, A\&A, 529, A163

Ohnaka, K., Hofmann, K.-H., Schertl, D., Weigelt, G., Baffa, C., Chelli, A., Petrov, R., \& Robbe-Dubois, S. 2013, A\&A, 555, A24

Schwarzschild, M. 1975, ApJ, 195, 137 